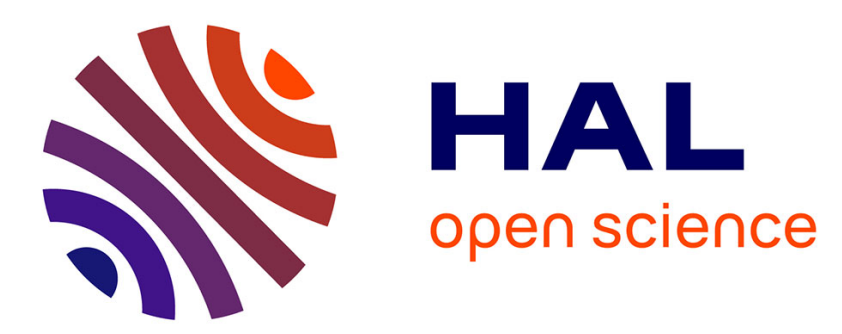

\title{
Optimal control of physical backwash strategy - towards the enhancement of membrane filtration process performance
}

Nesrine Kalboussi, Jérôme Harmand, Alain Rapaport, Térence Bayen, Fatma Ellouze, Nihel Benamar

\section{To cite this version:}

Nesrine Kalboussi, Jérôme Harmand, Alain Rapaport, Térence Bayen, Fatma Ellouze, et al.. Optimal control of physical backwash strategy - towards the enhancement of membrane filtration process performance. Journal of Membrane Science, 2018, 545, pp.38-48. 10.1016/j.memsci.2017.09.053 . hal-01591027

\section{HAL Id: hal-01591027 \\ https://hal.science/hal-01591027}

Submitted on 12 Jan 2018

HAL is a multi-disciplinary open access archive for the deposit and dissemination of scientific research documents, whether they are published or not. The documents may come from teaching and research institutions in France or abroad, or from public or private research centers.
L'archive ouverte pluridisciplinaire HAL, est destinée au dépôt et à la diffusion de documents scientifiques de niveau recherche, publiés ou non, émanant des établissements d'enseignement et de recherche français ou étrangers, des laboratoires publics ou privés. 


\title{
Optimal control of physical backwash strategy \\ - towards the enhancement of membrane filtration process performance
}

\author{
Nesrine Kalboussi a,b, Jérôme Harmand c, Alain Rapaport ${ }^{d}$, Térence \\ Bayen ${ }^{\mathrm{e}}$, Fatma Ellouze ${ }^{\mathrm{a}, \mathrm{b}}$, Nihel Ben Amar ${ }^{\mathrm{a}, \mathrm{b},{ }^{*}}$ \\ a Université de Carthage, Institut National des Sciences Appliquées et de Technologie, \\ B. P.676,1080 Tunis Cedex, Tunisia \\ b Université de Tunis El Manar, Ecole Nationale d'Ingénieurs de Tunis, Laboratoire de \\ Modélisation Mathématique et Numérique dans les sciences d'ingénieur, B. P 37 Le \\ Belvédère 1002 Tunis, Tunisia \\ c LBE, INRA, Univ Montpellier, 11100, Narbonne, France \\ d UMR INRA/SupAgro MISTEA, Montpellier, France \\ e Institut Montpelliérain Alexander Grothendieck, CNRS, Univ.Montpellier \\ * Corresponding author at: Laboratoire de Modélisation Mathématique et Numérique \\ dans les Sciences d'Ingénieur, Ecole Nationale d'Ingénieurs de Tunis, Université Tunis \\ El-Manar, BP 37, 1002, Tunis, Tunisie. Tél : +216 71871022 - Fax : +216 71871022 \\ E-mail addresses: \\ nesrinekalboussi@gmail.com (N. Kalboussi) \\ Jerome.Harmand@inra.fr (J. Harmand) \\ alain.rapaport@inra.fr (A. Rapaport) \\ terence.bayen@umontpellier.fr (T. Bayen) \\ ellouze_fatma@yahoo.fr (F. Ellouze) \\ benamar_nihel@yahoo.fr (N. Ben Amar)
}




\section{Keywords:}

Ultrafiltration

Microfiltration

Physical backwash strategy

Optimal control

Net water production optimization

\section{Introduction}

Membrane filtration technologies have shown great promise as physical separation techniques in the field of water treatment, including for example water desalination, wastewater treatment and drinking water production. It offers several benefits as compared to conventional treatment processes: better contaminant removal, smaller footprint and easy process automation [1]. However, the major drawback of a membrane filtration process remains membrane fouling which results over time in either an observable transmembrane pressure (TMP) increase under constant flux conditions or a dramatic drop in the permeate flux under constant pressure conditions [2].

Physical membrane cleaning at periodic intervals is one possible means for controlling and reducing fouling. Physical cleaning is a process that eliminates the reversible fouling from the membrane surface and pores. Commonly, it is carried out either by relaxation or backwash, or by a combination of both [3, 4]. Even though physical cleaning cannot eliminate long-term irreversible fouling phenomena, in the optimized conditions it can slow down the build-up of irreversible fouling, resulting in less frequent chemical cleaning, improvement in water productivity and reduction in the operating costs.

Membrane backwash is the reversal of flux back through the membrane. The effectiveness of such backwash in removing reversible fouling from the membrane surface and pores has been well studied $[5,6]$. Since the permeate itself is used as the medium for flushing the membrane, frequent backwashing will cause net 
production loss. However, infrequent backwashing increases the build-up rate of the internal fouling. The key factors for the optimization of the backwash operation are its flow rate, pressure, duration and frequency, along with the ratio between these two last parameters $[7,8]$. In practice, due to the complexity of the membrane filtration system these parameters are fixed according to the recommendations of the membrane manufactures or chosen according to the operator's experience [3]. This leads to suboptimal process performances, thereby wasting permeate and energy.

Several efforts to optimize the filtration and backwash periods have been made. In earlier work, Paul Chen et al. [9] presented a statistical approach using an overlay of surface response plots to optimize the filtration time between two backwash cycles. They effectively improved the system's production compared to the strategy suggested by the membrane supplier. However, it was recognized by the authors that further efforts are required to design more practical and cost-effective optimal operating strategies. In others papers, system performance optimization was obtained by developing an automatic control system for deciding on the cleaning action based on a comparison of the value of the controlled variable to a set-point value. For example, Smith et al. [10] developed a control system that monitors the TMP evolution over time and triggers a membrane backwash whenever the TMP exceeds a given set-point. Thus, backwash duration was automatically adjusted and the water required in membrane backwashing was reduced by $40 \%$ [10]. Similarly, the automatic control system presented in the work of Villarroel et al. [8] monitored the TMP to adjust the duration of relaxation and backwash. The results showed that the automatic control of membrane cleaning permitted an increase in filtration time and, thus, overall system productivity. On the other hand, Vargas et al. [11] used the permeate flux as the controlled variable to automatically control the membrane backwash in a submerged membrane bioreactor. Backwash frequency was increased if the permeate flux dropped to the minimum allowable set-point.

Mathematical modeling is an effective tool for developing control strategies designed to control fouling and, thus, to optimize membrane filtration process performance. Drews et al. [12] presented a model-based control strategy that gathered flux data and identified the current state of membrane fouling in order to take the appropriate response (aeration, relaxation or backwash). With respect to knowledge-based control of membrane filtration, Robles et al. $[13,14]$ developed a controller that aimed at 
reducing energy consumption and system downtime due to membrane backwash. Recently, Cogan et al. [15, 16], using a simple specific model of ultrafiltration/microfiltration processes, adopted an analytical approach applying Pontryagin's Maximum Principle to predict the optimal instants of switching between backwash and filtration periods that maximize the overall water production in a given time frame. However, Cogan et al.'s optimal control analysis is questionable because the transversality condition of the Pontryagin Maximum Principle was not verified. In addition, the solution proposed was only applicable to very specific initial conditions, assuming a completely clean membrane.

In a previous work [17], we solved the problem of optimizing the total production of a membrane filtration process operating at constant TMP and including periodic relaxation between filtration cycles. The analytical solution to this optimization problem was given. The resulting optimal control strategy was able to improve the total volume filtrated by about $76 \%$ compared to the classic operating strategy.

As in [17], the present work is devoted to the study of an analogous optimal control problem. However, membrane unclogging is performed here by periodic cycles of backwash instead of membrane relaxation. The main goal of this paper is thus to determine the optimal switching between filtration and backwash that mitigates fouling while maximizing over a given period of time the net water production per membrane area of a membrane filtration process operating at constant TMP.

The paper is organized as follows: in the first section, the optimal solution to the optimization problem is given for two case studies: (i) when the backwash flux is constant; and (ii) when the backwash flux depends on the degree of fouling. We will show that in both cases, the general pattern of the analytical solution is the same: it does not depend on any specific mathematical model. To demonstrate the effectiveness of the proposed control strategy, we consider a specific case for which a mathematical model is calibrated on the basis of published experimental data. Only the results of model validation and parameter identification are shown insofar as the main purpose of this work is to present the optimal control strategy for a membrane filtration system. Because the optimal solution appears to be unfeasible in practice, we propose an approximation approach to adapt it to the real constraints of a membrane filtration process. Finally, we study the robustness of the optimal control strategy with 
respect to various disturbances that are actually present when dealing with any pilotor industrial-scale membrane separation process.

\section{Optimal operation strategy}

\subsection{Statement of the optimal control problem}

In this section, we introduce the optimal control problem for a membrane filtration process running at a constant TMP. The process works by alternating two functional modes: filtration and physical backwash. An optimal control problem consists in searching for a control signal such that an objective function is minimized or maximized, given a mathematical model representing the dynamics of the process involved and possible constraints. Here, the objective is the mitigation of membrane fouling in order to maximize the total filtered water obtained over a given period of time $T$. In order to derive generic solutions to the problem, rather than considering a specific mathematical model, we use a class of filtration models defined only by their qualitative behaviors.

Depending on the assumption made about the backwash flux, two different cases are studied below: (i) when the backwash flux is considered constant and equal to an average value; and (ii) when it varies depending on the extent of fouling.

When a membrane operates in filtration, the resistance to flow increases according to the mass of the cake layer formed on the membrane surface, termed $m$, which subsequently decreases the permeate flux. Thus, we assume that the rate at which the mass of material adheres to the membrane surface during filtration is a positive decreasing function such that the dynamics of mass attachment is given by equation (1):

$$
\dot{m}=f_{1}(m)>0
$$

On the other hand, when starting membrane backwash, the cake layer is decomposed and the membrane's permeability increases again. So, the dynamics of the cake detachment can be described by an increasing negative function given by:

$$
\dot{m}=-f_{2}(m) \leq 0
$$


We can write a hybrid model that combines these two dynamics into a single equation through the control variable $u(t)$ taking, by convention, the value 1 during filtration mode and -1 during membrane backwash:

$$
\dot{m}=\frac{1+u(t)}{2} f_{1}(m)-\frac{1-u(t)}{2} f_{2}(m)
$$

At constant TMP, the permeate flux decreases as the extent of fouling gradually increases. Therefore, the variation of the permeate flux, $J$, can be described by a decreasing positive function of the mass of the fouling layer:

$$
J(m)>0
$$

In the next section, the optimization method used to determine the optimal operating strategy of a membrane filtration system is described briefly, the main results are summarized and details are given in the Appendices A and B.

\subsection{Optimization criteria}

In case \#1, we consider that membrane backwash is performed with a constant flux and that backwash pressure varies depending on the extent of fouling. In case \#2, we assume that during backwash the pressure is constant and the backwash flux increases as soon as the membrane has been regenerated. The key difference between these two cases lies in the objective function to be maximized. In both cases, Pontryagin Maximum Principle (PMP) [18] is the mathematical tool applied to determine the analytical solution of the optimal control problem.

\section{- Case \#1: Operating with constant backwash flux}

The criterion to be optimized is given by Eq.(5) below. In fact, the net water production per membrane area of the process during an overall time interval $[0, T]$ is the total permeate volume produced during filtration per membrane area minus the total permeate volume used during backwash periods per membrane area.

$$
L_{T, 1}=\int_{0}^{T}\left(\frac{1+u(t)}{2} J(m(t))-\frac{1-u(t)}{2} J_{B W}\right) d t
$$

where $J(m(t))$ and $J_{B W}$ are respectively the permeate flux and the backwash flux. 


\section{- Case \#2: Operating with variable backwash flux}

If during membrane backwash, the backwash flux varies depending on the amount of fouling removed, then the functional cost of the optimal control problem is given by:

$$
L_{T, 2}=\int_{0}^{\mathrm{T}} u(t) J(m(t)) d t
$$

where $J(m(t))$ is the forward or backward permeate flux such that $L_{T, 2}$ increases during filtration but decreases during backwash.

\subsection{The optimal solution}

The mathematical analysis shows (cf. Appendices A and B) that the optimal solution is similar in both cases: the optimal control depends on the state of membrane fouling $m(t)$ which is given by:

$$
u^{*}(t, m)=\left\{\begin{array}{rr}
1, \quad \text { if } m<\bar{m} \text { or } t \geq \bar{T} \\
-1, \quad \text { if } m=\bar{m} \text { and } t<\bar{T}
\end{array}\right.
$$

where the control parameters, $\bar{m}, \bar{u}$ and $\bar{T}$, are computed using the PMP. In other words, in both cases and over a given period of time $[0, T]$ the optimal strategy can be summarized as follows: in a first phase, $\left[0, t_{e}\right]$, if the initial mass attached to the membrane, $m(0)$, is less than a given value $\bar{m}$, the process operates in filtration (i.e. $u=1$ ) until $m$ reaches $\bar{m}$. Otherwise, if the membrane is already significantly fouled (i.e. $m(0)>\bar{m}$ ), a backwash is necessary to regenerate the membrane until the state $m$ equals $\bar{m}$. Then, in a second phase, $\left[t_{e}, \bar{T}\right]$, a constant control $\bar{u}$, given a value between -1 and 1 , is applied to maintain the mass of the cake layer around $\bar{m}$ until the switching time $\bar{T}$ is reached. Finally, when the operating time attains $\bar{T}$, it is optimal to finish the process run cycle with filtration $(u=1)$ until the final time, $T$.

As shown, mass accumulation on the membrane surface cannot be avoided. However, the proposed optimal control strategy keeps $m$ around a "threshold" value $\bar{m}$ and thus permits the mitigation of fouling effects on process performance. It is almost like the critical flux concept introduced by Field et al. [19]. The critical flux is defined as the flux below which the process can continue to operate over long time periods with negligible membrane fouling. 
Remark 1: Note that the control parameters $\bar{m}, \bar{T}$ and $\bar{u}$ depend only on the parameters of the fouling dynamics in the model. In other words, they can be computed once and the optimal control (i.e. Eq.(7)) can be applied whatever the initial condition.

Remark 2: All values of the control parameters $\bar{m}, \bar{T}$ and $\bar{u}$ will be stated later for a specific filtration model identified from real data. The key strength of the approach presented here is that it provides a generic solution based solely on the qualitative properties of membrane fouling dynamics. Thus, the general pattern presented in Eq.(7) depends exclusively on the general assumptions made for expressions (1) to (4).

Remark 3: The value $\bar{u}$ is called a "singular control" (cf. Appendices A and B). Since the control involves $\bar{u}$ which takes a value between -1 (i.e. backwash) and 1 (i.e. filtration), the analytical solution of the optimization problem presented here cannot be applied in practice; it has no physical meaning. In section 5, this problem is addressed and an approximation approach is provided to adapt the analytical solution to the practical considerations. Basically, the singular control $\bar{u}$ is approximated by a periodic switching of filtration $(u=1)$ and membrane regeneration $(u=-1)$, such that the fouling mass $m(t)$ remains close to $\bar{m}$ during the time interval in which it is optimal to apply $\bar{u}$.

Remark 4: It should also be noted that the total operating time of the process, $T$, only affects $\bar{T}$. Consequently, if we solve the problem for a large $T$ the values of the control parameters $\bar{m}, \bar{u}, \bar{m}_{T}$ and $t_{e}$ remain unchanged as for a short $T$. However, the last moment for switching to filtration, $\bar{T}$, occurs later because the duration of the first and last filtration cycle does not change.

\section{Materials and methods}

\subsection{Mathematical filtration model}

In the previous section, we presented the optimal operating strategy that maximizes the net water production per membrane area of a membrane filtration process over a given time $T$.

At this point, in order to evaluate its effectiveness, the proposed solution is applied in a simulation case-study described by a specific mathematical model. Care has been 
taken to consider a simple model in order to ensure that the control parameters can be expressed analytically.

The filtration model considered assumes that membrane fouling is due only to cake layer formation. Moreover, it takes into account the effects of backwash cleaning by reversing the permeate flux through the membrane. Many studies have shown that models of cake blocking appear most suited to predicting flux decline (or TMP increase), especially during ultrafiltration or when using high suspended-solids concentrations [20-23].

The model considered here establishes that the cake layer mass, $m$, grows in proportion to the concentration of foulants in the feed, $C_{\text {tot }}$, and to the permeate flow passing through the membrane, $Q_{P}$.

$$
\dot{m}=f_{1}(m)=Q_{P} C_{t o t}
$$

The variation in permeate flux is expressed based on the resistance-in-series concept. In fact, the evolution over time of the permeate flux depends on the total filtration resistance $R_{\text {tot }}$, which is the sum of the membrane's intrinsic resistance $R_{0}$ and the cake layer resistance $R_{c}$. The cake layer resistance is proportional to the mass $m$ accumulated on the membrane surface as given by the equation (9):

$$
R_{c}=\frac{\alpha}{A} m
$$

where $\alpha$ is the specific resistance of the cake layer $\left(m \cdot \mathrm{kg}^{-1}\right)$ and $A$ is the membrane surface $\left(m^{2}\right)$. By replacing the equation Eq. (9) in the Darcy law, $m$ appears in the flux variation $J(m)$, such that:

$$
J(m)=\frac{Q_{p}}{A}=\frac{T M P}{\mu\left(R_{0}+\frac{\alpha}{A} m\right)}
$$

Henceforward, the model equations will be presented in a simplified form as functions of the parameters $b, d, e, a_{1}$ and $a_{2}$. The physical significance of these parameters in relation to the corresponding functions is shown in Table 1. Therefore, Eq.(8) and (10) can be written as: 


$$
\begin{gathered}
\dot{m}=f_{1}(m)=\frac{b}{e+m} \\
J(\mathrm{~m})=\frac{d}{e+m}
\end{gathered}
$$

When we assume that the backwash flux is constant (i.e. case \#1), the mass detaches from the membrane surface as a function of the constant backwash flux, $J_{B W}\left(\mathrm{~m}^{3} \cdot \mathrm{m}^{-2}\right.$. $\left.\mathrm{h}^{-1}\right)$, and the detachment resistance of foulants, $\omega_{B W}\left(m^{-1}\right)$, such that:

$$
\dot{m}=-f_{2}(m)=-\omega_{B W} J_{B W} m=-a_{1} m
$$

For the other case (i.e. case \#2), when the backwash flux depends on the current membrane fouling, the removal of solids from the membrane surface is described by:

$$
\dot{m}=-f_{2}(m)=-\omega_{B W} J(m) m=-a_{2} \frac{d}{e+m} m
$$

This mathematical model is used as an application to demonstrate the effectiveness of our generic optimal control solution in optimizing the production of a membrane filtration process. In addition, it should be noticed that any other model can be used, provided that it meets the general assumptions made in section 2.1.

In the next section, we will estimate the model parameters from published data in order to evaluate the parameters of the optimal control solution (i.e. $\bar{m}, \bar{T}, \bar{u}$ ) for a specific application.

\subsection{Parameter estimation}

To estimate the parameters of the simplified model, we extracted data from previous experiments published in the work of Xia et al. [1]. In this work, the authors investigated the use of ultrafiltration hollow fiber membranes to treat polluted reservoir water in China for drinking water production. To this end, specific tests were conducted to evaluate the variation of the permeate flux as a function of different operating parameters such as backwash strategy. The authors showed that performing a backwash cycle for $40 \mathrm{~s}$ after a filtration cycle of $60 \mathrm{~min}$ can restore permeate flux to initial levels. The permeated water was used to backwashing the membrane and then the used water was discharged from the recirculation loop. 
The net water production per membrane area of a membrane filtration process using Xia et al.'s [1] strategy will be compared to ours. Henceforward, we refer to the Xia et al.'s strategy as the "classic" strategy.

Data were extracted using free software, WebPlotDigitizer, from a plot describing permeate flux variation published in [1]. WebPlotDigitizer is a simple tool used to identify and extract the coordinates of each point from an uploaded image of the graph. The extraction of the data points comes after calibrating the axis by assigning four points of known values on the axis. Obviously, the extracted numerical data may be a little biased by this procedure, but what is most important is that the model is able to describe their global dynamics very well. In each case, these data will be used to find the optimal set of model parameters that best reproduce them.

The optimization of the model's parameters was done using the optimization function FminSearch of MATLAB in such a way that the simulated data best matched the extracted data when minimizing a least-square criterion. Since the only difference between the two case studies was the backwash dynamics, the parameters of the filtration dynamics were the same in both cases.

The values of the optimized model parameters of case \#1 and \#2 (i.e. $b, e, d, a_{1}, a_{2}$ ) are reported in Table 2. Henceforward, these sets of parameters are used for all the numerical simulations of the optimal control solution. The fittings between the experimental and the simulated permeate fluxes in each case are represented in Fig.1. Despite its simplicity, the model reproduces in a satisfactory way the experimental data. In order to evaluate the fitting efficiency, the coefficients of the determination $R^{2}$ have been calculated and are shown in Fig. 1 . The $R^{2}$ value in case \#2 is slightly smaller than that obtained in case $\# 1$. However, in both cases, $R^{2}$ is greater than $88 \%$.

\section{Optimization results}

For each case, the appropriate mathematical model with the parameters identified were used to calculate the optimal control parameters. Simulations were performed using MATLAB for a time period T of 7 hours which was the same as in the study from which experimental data were extracted. In case \#1, the backwash flux is twice the 
initial permeate flux. For both cases, the values of the optimal control parameters are given in Table 3.

Considering the model as a "virtual process", we can now choose any initial condition, for instance $m(0)=0$, and apply the theoretical control to the model dynamics given by Eq.11-14 for both cases. Figs. 2 and 3 show the theoretical optimal control solutions respectively, for case \#1 when the backwash flux is considered constant and for case \#2 when the backwash flux is a function of the detached mass. In both cases, since we began with a clean membrane (i.e. $m(0)=0$ ), the process was allowed to operate in filtration for about $11 \mathrm{~min}$ in case \#1 and $6 \mathrm{~min}$ in case \#2 before the amount of fouling reached $\bar{m}$. The first filtration cycle lasted longer in case \#1 than in case \#2 because, as shown in Table 3 , the value of $\bar{m}$ was bigger in case \#1 than in case \#2. Then, $u$ was set equal to $\bar{u}=0.9$ in case \#1 and 0.87 in case \#2. In both cases, $\bar{u}$ was positive and close to 1 . When the singular control $\bar{u}$ was applied, the mass of the cake layer was kept constant at $\bar{m}$. The final filtration cycle lasted $7 \mathrm{~min}$ for case \#1 and $8 \mathrm{~min}$ for case \#2. We note that the length of the first and the final filtration cycles are short compared to the time spent on the singular arc defined by $m(t)=\bar{m}$. Consequently, to reduce membrane fouling it is important that the state $m$ reaches $\bar{m}$ as quickly as possible and remains there as long as possible.

When applying the Xia et al.'s strategy, the net water production per membrane area over 7 hours was 918.32 L.m $\mathrm{m}^{-2}$. As expected, our optimal strategy significantly enhanced the maximum net production per membrane area of the membrane filtration process, as shown in Fig.4. When the backwash flux is constant, the optimal solution improves the maximum net production per membrane area of the process by $18.5 \%$, compared to the "classic" strategy published in [1]. When the backwash flux depends on the extent of fouling, the optimal solution leads to an even greater improvement of $33 \%$ when compared to the strategy in [1].

\section{Practical implementation}

Since a control $\bar{u}$ different to 1 or -1 has no physical meaning, the question now is to approximate $\bar{u}$ by only filtration and backwash cycles, such that $m(t)$ remains close to $\bar{m}$ during the time interval corresponding to the singular arc. This approach is known in the literature as a chattering control [24, 25]. Notice that applying such an 
approximation strategy will necessarily degrade the control performances since, by definition, any control signal will be less effective than the optimal one.

Although various strategies can be proposed, in a first approach we considered that $\bar{u}$ is the percentage of an operating cycle time spent in filtration, such that:

$$
\begin{gathered}
\frac{T_{f}}{T_{p}}=\frac{1+\bar{u}}{2} \\
T_{p}=\frac{T_{S A}}{N}=T_{f}+T_{B W}
\end{gathered}
$$

where $T_{f}$ and $T_{B W}$ are the filtration and the backwash times during an operating cycle, $T_{p}$ is the time of an operating cycle, $T_{S A}$ denotes the total time for which it is optimal to apply the constant control $\bar{u}$ and $N$ is the number of cycles over the time-period $T_{S A}$. Carried to the extreme, the latter expression means that if $\bar{u}=1$, then all the cycle time will be spent in filtration $\left(T_{f}=T_{p}\right)$. At the other extreme, if $\bar{u}=-1$, then all the cycle time will be spent in backwash $\left(T_{f}=0\right)$.

Figs. 5 and 6 show what happens when we approximate the singular control by applying $N=20$ cycles. In both cases, the mean accumulated mass on the membrane surface is relatively close to $\bar{m}$ and the maximum net volume per membrane area produced approaches the level of optimal production.

Here, a possible question may arise: what would be the impact of increasing the switching frequency on control performance while keeping the average of filtration time over an operating cycle (i.e. $\bar{u}$ ) constant? To answer this question, we varied the number of cycles $N$ between 2 and 100 and plotted the maximum net production per membrane area of the process over $T$ as a function of $N$ (see Fig.7). As the number of switches $N$ increased, the mass of the cake layer tended towards $\bar{m}$ and process production over $T=7$ hours was significantly improved: when $N=100$, it reached a value close to the optimal theoretical production. In other terms, the closer the fouling mass to $\bar{m}$ on the singular arc, the better the performance. It should be noted that even with a small $N(5<N<10)$ the mean accumulated mass on the membrane surface was not too far from $\bar{m}$ (c.f. Fig.7). Moreover, further increases $N$ does not yield any significant gain: $98 \%$ of the optimal production is reached for $\mathrm{N}=30$ cycles of 13 min in case \#1 and $\mathrm{N}=40$ cycles of $10 \mathrm{~min}$ in case \#2 (c.f. Fig.7). In fact, if the frequency of 
switching becomes too great, the risk of wear to the permeation pumps increases. It is thus self-evident that the optimal control when applied in practice is a compromise between the number of switches and the practical constraints related to using the pumps. An open question for the future is to establish whether or not there exists a better approximation strategy, for example utilizing less energy while maintaining a high performance.

\section{Robustness analysis}

This section studies the behavior of the proposed optimal control solution with respect to uncertainty in model parameters. Robustness refers to the ability of the optimal control to maintain any solution close to the optimum in the presence of disturbances and/or uncertainty about model parameters. It is obvious that modeling errors as well as disturbances in real membrane filtration processes are unavoidable. They may lead to increasing divergence between the real and the simulated-state variables. In fact, modeling membrane separation systems entails certain difficulties because of the unmeasurable nature of the process state such that the kinetics of membrane fouling make it difficult to properly identify model parameters. For example, disturbances in a real membrane filtration process may be attributed to variations in feed quality, feed pressure or membrane properties, all of which can affect a model in real time.

As a consequence, it is important to evaluate the performance of a control that has been designed with specific model parameters when it is actually applied to a process with slightly different dynamics. To do so, the following approach has been considered: we start by introducing random biases of $\pm 20 \%$ in the model's parameters in order to generate a set of disturbed systems. Then the optimal solution, computed using the parameters of the nominal system, is applied to the disturbed process. To measure robustness, the deviation of the maximum net production per membrane area from the optimum is calculated for each model, according to the following index:

$$
\gamma(\%)=\frac{\left(L_{p}-L_{o p t}\right)}{L_{o p t}} * 100
$$

where $\gamma$ is the percentage of deviation from the optimal net water production per membrane area $L_{o p t}$, while $L_{p}$ is the net water production per membrane area of the disturbed system. 
Out of 1000 iterations, the results given in Table 4 make clear that, in both cases, if the disturbances are added to parameters $a_{1}, a_{2}$, and $b$, the deviation in the net water production per membrane area never exceeds $5 \%$ in absolute value. However, when the disturbances occur in parameters $d$ and $e$, which are directly dependent on the permeate flux equation, the divergences between the production of the nominal and the disturbed systems become greater. The highest mean deviation, approximately $22.5 \%$ (in absolute value), is observed in both cases when parameter $d$ is disturbed. Similarly, if the disturbance is introduced in parameter $e$, the divergence of the production from the optimum ranges from $-13.6 \%$ to $17.06 \%$ in case \#1 and from $14.65 \%$ to $20.76 \%$ in case \#2. Thus, in the worst cases for the example we have presented, the deviation from the optimal can be of the order of the gain observed when applying the optimal strategy (cf. section 4). This means that great care must be taken at the identification stage and that efforts are needed in identifying good models.

\section{Discussions}

In real MF/UF processes, membrane fouling mechanisms are multiple and complex. The contribution of each mechanism in the filtration process performances decline depends on the membrane-particles and particles-particles interactions, which in turn are related to the membrane properties (membrane material, pore size, pore size distribution, membrane hydrophobicity), the effluent characteristics (type: biofoulants, organic and inorganic foulants; particles size and concentration), the operating conditions (flow rate, temperature, pressure, $\mathrm{pH}$ ) [26, 27] and the filtration mode (deadend filtration, cross-flow filtration). The novelty of the present work is that the determined optimal solution is generic and it can easily be adapted to deal with the specificity of the filtration process in question. This is done by considering the appropriate fouling mechanism that best describes the system provided that the qualitative assumptions made on the fouling dynamics during filtration and backwashing phases hold.

Obviously, the proposed approach relies on the use of a model that correctly captures filtration and backwash dynamics under specific operation conditions. If these conditions change, the model has to be adjusted and the control re-synthesized. Then, it is essential to identify and validate the defined model based on real data acquired on the process under conditions of interest. 
If the dynamics of the considered model can be captured using the simple model assumptions introduced in this work, then the proposed optimal solution can be computed and applied.

Another important point to note that we actually do not need to measure $m$ neither for identifying the model of the system nor for applying the optimal control. Regarding the identification of the models, we simply need to characterize filtration and backwash dynamics (functions $f_{1}, f_{2}$ and $J$ ) which can be done using only the measurement of the permeate flux. Concerning the control, there are many ways of applying the control on a real process. To obtain the best results, the control should be implemented in closed loop (c.f. Fig.8). However, as such, it requires the measurement of $m$ which is not feasible in practice. Whatever the initial state of the membrane, this limit could be overcome by the online measurement of the permeate flux which allows the determination of the instantaneous value of $m$ using the permeate flux function $J(m)$ presented in Eq.10.

Another possibility is to apply the control in open loop (c.f. Fig.8), without any feedback (i.e. the online knowledge of $m$ ). In this case, we only need to determine the initial value of $m$. If the membrane is clean at the beginning of the experiment, the user must rely on the model identified to know exactly how long he must apply $u=1$ to reach $m=\bar{m}$ and then run the alternation of filtration and backwash periods. If the membrane is not clean and $m_{0}>\bar{m}$, the operator has to initiate a backwash cycle until the calculated time $t_{e}$ is reached and then he applies a determined number of switches between filtration and backwash cycles. Finally, under the assumption that $m$ is close to $\bar{m}$, we can simply alternate filtration and backwash periods with the optimal ratio computed from the knowledge of $\bar{u}$. Notice also that if the control is applied in open loop, it is not possible to take into account the effect of unexpected disturbances that can decrease the control efficiency.

\section{Conclusions}

This study shows the benefits of the application of the Pontryagin Maximum principal in optimizing the net water production per membrane area of a membrane filtration system operating at constant TMP. The main advantage of the optimal solution proposed here is that it has been designed for a very large class of models. In other 
words, its general pattern remains similar as long as very general assumptions on process dynamics, defined as the qualitative properties of a fouling model, hold. The solution has been given for two different cases, depending on whether the backwash flux is constant or variable. The application in simulation of the optimal strategy to a specific case study significantly improves the net production per membrane area compared to a classic strategy. Moreover, we show that is possible to adapt the analytical solution for practical application by increasing the frequency of switching on the singular arc. The cost related to the amount of switching between filtration and backwash modes is not taken into account here but will be the subject of our future work. The perspectives of this work also include the evaluation of the optimal solution on laboratory-scale membranes and the extension of the approach developed here to tackle the problem of minimizing the process's energy consumption when run with constant flux. It is important also to evaluate the proposed optimal solution on laboratory-scale membranes in order to conclude about the performances and the robustness of the controller in practice.

The synthesis of the optimal solution in the present work was based on a mathematical framework that consider the predominance of only one fouling mechanism. In the future, we plan to consider combined models that take into account the presence of different fouling mechanisms (such as cake formation and pores clogging). Moreover, as in practice membrane flux recovery is often not performed only by backwash, but rather by the combination of backwash and relaxation periods (with or without aeration), the detachment effect of the relaxation with backwashing will be considered and the same approach will be applied to determine the optimal solution for such class of MF/UF processes.

Acknowledgements: The authors thank the Euromed research network TREASURE (www.inra.fr/treasure), the French Institute of Tunis (www.institutfrancais-tunisie.com) and Adalberto Noyola for the fruitful discussions we had about this work. 


\begin{tabular}{|c|c|}
\hline \multicolumn{2}{|l|}{ Nomenclature } \\
\hline$A$ & Membrane surface $\left(m^{2}\right)$ \\
\hline$C_{\text {tot }}$ & Total suspended solids concentration $\left(g \cdot L^{-1}\right)$ \\
\hline$J$ & Permeate flux $\left(L . h^{-1} \cdot m^{-2}\right)$ \\
\hline$J_{B W}$ & Backwash flux $\left(L \cdot h^{-1} \cdot m^{-2}\right)$ \\
\hline$L_{\text {opt }}$ & Optimal net water production per membrane area $\left(L \cdot m^{-2}\right)$ \\
\hline$L_{p}$ & Net water production per membrane area of the disturbed system \\
\hline & $\left(L . m^{-2}\right)$ \\
\hline$L_{T}$ & Net water production per membrane area $\left(L . m^{-2}\right)$ \\
\hline $\bar{m}$ & value of the fouling mass on the singular arc $(g)$ \\
\hline $\bar{m}_{T}$ & Fouling mass at the final time $(g)$ \\
\hline$N$ & Number of cycles over the time-period $T_{S A}$ \\
\hline$Q_{P}$ & Permeate flow $\left(L . h^{-1}\right)$ \\
\hline$R_{0}$ & Membrane's intrinsic resistance $\left(m^{-1}\right)$ \\
\hline$R_{c}$ & Cake layer resistance $\left(m^{-1}\right)$ \\
\hline$T$ & Total operating time of the process $(h)$ \\
\hline$T M P$ & Trans-Membrane Pressure $(\mathrm{Pa})$ \\
\hline$T_{B W}$ & Backwash time during an operating cycle $(h)$ \\
\hline$t_{e}$ & First switching time $(h)$ \\
\hline$T_{f}$ & Filtration time during an operating cycle $(h)$ \\
\hline$T_{p}$ & Time of an operating cycle $(h)$ \\
\hline$T_{S A}$ & Total time of the singular arc $(h)$ \\
\hline $\bar{T}$ & Final switching time $(h)$ \\
\hline$u$ & Control variable \\
\hline$u^{*}$ & Optimal control \\
\hline $\bar{u}$ & Singular control \\
\hline$\omega_{B W}$ & Detachment resistance of foulants $\left(\mathrm{m}^{-1}\right)$ \\
\hline$\alpha$ & Specific resistance of the cake layer $\left(\mathrm{m} . \mathrm{kg}^{-1}\right)$ \\
\hline$\mu$ & Permeate viscosity (Pa.s) \\
\hline
\end{tabular}




\section{References}

1. [data] Shengji, X., et al., Application of membrane techniques to produce drinking water in China. Desalination, 2008. 222(1-3): p. 497-501.

2. Miller, D.J., et al., Comparison of membrane fouling at constant flux and constant transmembrane pressure conditions. Journal of Membrane Science, 2014. 454: p. 505-515.

3. Judd, S., The MBR book: principles and applications of membrane bioreactors for water and wastewater treatment. 2010: Elsevier.

4. Yusuf, Z., N. Abdul Wahab, and S. Sahlan, Fouling control strategy for submerged membrane bioreactor filtration processes using aeration airflow, backwash, and relaxation: a review. Desalination and Water Treatment, 2016. 57(38): p. 17683-17695.

5. Psoch, C. and S. Schiewer, Critical flux aspect of air sparging and backflushing on membrane bioreactors. Desalination, 2005. 175(1): p. 61-71.

6. Psoch, C. and S. Schiewer, Resistance analysis for enhanced wastewater membrane filtration. Journal of membrane science, 2006. 280(1): p. 284-297.

7. Le-Clech, P., V. Chen, and T.A. Fane, Fouling in membrane bioreactors used in wastewater treatment. Journal of membrane science, 2006. 284(1): p. 17-53.

8. Villarroel, R., et al., Physical cleaning initiation controlled by transmembrane pressure set-point in a submerged membrane bioreactor. Separation and Purification Technology, 2013. 104: p. 55-63.

9. Chen, J.P., S. Kim, and Y. Ting, Optimization of membrane physical and chemical cleaning by a statistically designed approach. Journal of Membrane Science, 2003. 219(1): p. 27-45.

10. Smith, P.J., et al., A new approach to backwash initiation in membrane systems. Journal of Membrane Science, 2006. 278(1): p. 381-389.

11. Fully-automatic control nanofiltration membrane high-flux flushing device. 2015, Google Patents.

12. Drews, A., et al., Model-based recognition of fouling mechanisms in membrane bioreactors. Desalination, 2009. 236(1): p. 224-233.

13. Robles, A., et al., Model-based automatic tuning of a filtration control system for submerged anaerobic membrane bioreactors (AnMBR). Journal of Membrane Science, 2014. 465(0): p. 1426.

14. Robles, A., et al., Advanced control system for optimal filtration in submerged anaerobic MBRs (SAnMBRs). Journal of Membrane Science, 2013. 430(0): p. 330-341.

15. Cogan, N. and S. Chellam, A method for determining the optimal back-washing frequency and duration for dead-end microfiltration. Journal of Membrane Science, 2014. 469: p. 410-417.

16. Cogan, N., et al., Optimal backwashing in dead-end bacterial microfiltration with irreversible attachment mediated by extracellular polymeric substances production. Journal of Membrane Science, 2016. 520: p. 337-344.

17. Kalboussi, N., et al., Optimal control of a membrane filtration system, in IFAC'2017. 2017: Toulouse, France.

18. Gamkrelidze, R., L.S. Pontrjagin, and V.G.e. Boltjanskij, The mathematical theory of optimal processes. 1964: Macmillan Company.

19. Field, R., et al., Critical flux concept for microfiltration fouling. Journal of Membrane Science, 1995. 100(3): p. 259-272. 
20. Charfi, A., N.B. Amar, and J. Harmand, Analysis of fouling mechanisms in anaerobic membrane bioreactors. Water research, 2012. 46(8): p. 2637-2650.

21. Waeger, F., T. Delhaye, and W. Fuchs, The use of ceramic microfiltration and ultrafiltration membranes for particle removal from anaerobic digester effluents. Separation and Purification Technology, 2010. 73(2): p. 271-278.

22. Ho, J. and S. Sung, Effects of solid concentrations and cross-flow hydrodynamics on microfiltration of anaerobic sludge. Journal of Membrane Science, 2009. 345(1): p. 142-147.

23. Wu, J., et al., Effects of relaxation and backwashing conditions on fouling in membrane bioreactor. Journal of Membrane Science, 2008. 324(1): p. 26-32.

24. Zelikin, M.I. and V.F. Borisov, Theory of chattering control: with applications to astronautics, robotics, economics, and engineering. 2012: Springer Science \& Business Media.

25. Bayen, T. and F. Mairet, Minimal time control of fed-batch bioreactor with product inhibition. Bioprocess and biosystems engineering, 2013. 36(10): p. 1485-1496.

26. Iorhemen, O.T., R.A. Hamza, and J.H. Tay, Membrane Bioreactor (MBR) technology for wastewater treatment and reclamation: membrane fouling. Membranes, 2016. 6(2): p. 33.

27. Rezaei, H., F.Z. Ashtiani, and A. Fouladitajar, Fouling behavior and performance of microfiltration membranes for whey treatment in steady and unsteady-state conditions. Brazilian Journal of Chemical Engineering, 2014. 31(2): p. 503-518.

\section{Appendix A. Case \#1: Operating with constant backwash flux}

The objective is to determine the optimal control $u($.$) that maximizes the net water$ production per membrane area of the membrane filtration process, $L_{T}$, over a given period of time $[0, T]$. The total water production per membrane area of the process is expressed as:

$$
L_{T}=\int_{0}^{T}\left(\frac{1+u(t)}{2} J(m(t))-\frac{1-u(t)}{2} J_{B W}\right) d t
$$

The total production per membrane area $L_{T}$ is subject to the dynamics of the membrane fouling given by:

$$
\dot{m}=\frac{1+u(t)}{2} f_{1}(m)-\frac{1-u(t)}{2} f_{2}(m)
$$

where $f_{1}(m)$ is the velocity of mass accumulation during filtration and $f_{2}(m)$ is the velocity of mass detachment during backwash. In order to provide a generic solution, we define the model functions only by their qualitative properties, such that:

i. $\quad f_{1}$ and $J$ are positive decreasing functions with $\lim _{m \rightarrow \infty} J(m)=0$

ii. $f_{2}$ is a positive increasing function with $f_{2}(0)=0$ 
For convenience, we define:

$$
J_{+}(m)=\frac{J(m)+J_{B W}}{2} ; J_{-}(m)=\frac{J(m)-J_{B W}}{2} ; f_{+}(m)=\frac{f_{1}(m)+f_{2}(m)}{2} ; f_{-}(m)=\frac{f_{1}(m)-f_{2}(m)}{2}
$$

The Hamiltonian associated to the cost function (Eq.(A.1)) and the filtration model (Eq.(A.2)) is given by:

$$
H(m, \lambda, u)=\lambda f_{-}(m)+J_{-}(m)+u\left[J_{+}(m)+\lambda f_{+}(m)\right]
$$

where the term multiplied by the control variable $u$ is called the "switching function" $\varphi$ (Eq. A.4)). Roughly speaking, the switching function determines when the control must be switched between the different possible values of the control variable (i.e. for instance, filtration or backwash but also, possibly, from one of these modes and a singular arc):

$$
\varphi(m, \lambda)=J_{+}(m)+\lambda f_{+}(m)
$$

According to the PMP, the optimal solution $u_{o p t}(t)$ that maximizes the objective function of the optimization problem has to maximize the Hamiltonian, such that:

$$
u_{o p t}(m, \lambda)=\left\{\begin{array}{c}
1 \text { when } \varphi(m, \lambda)>0 \\
-1 \text { when } \varphi(m, \lambda)<0
\end{array}\right.
$$

In the Hamiltonian expression (Eq.(A.3)), $\lambda$ is the adjoint variable that satisfies the terminal condition, $\lambda(\mathrm{T})=0$, and the following differential equation:

$$
\dot{\lambda}=-\frac{\partial H}{\partial m}=-\left[\lambda f_{-}^{\prime}(m)+J_{-}^{\prime}(m)+u\left[J_{+}^{\prime}(m)+\lambda f_{+}^{\prime}(m)\right]\right]
$$

At the terminal time $T$, we obtain $\varphi(m(T), \lambda(T))=J_{+}(m(T))>0$. By continuity, the function $\varphi$ is positive on a time interval $[\bar{t}, T]$ with $\bar{t}<T$. Thus it is optimal to operate in filtration mode $(u=1)$ on this time interval.

Now, we check the existence of a singular arc in the time interval before the instant $\bar{t}$. On a singular arc, one has $\varphi=0$ and $\dot{\varphi}=0$. Let us write the derivative of the switching function : 


$$
\begin{gathered}
\dot{\varphi}=\left(J_{+}^{\prime}+\lambda f_{+}^{\prime}\right)\left(u f_{+}+f_{-}\right)+f_{+}\left[-\lambda f_{-}^{\prime}-J_{-}^{\prime}-u\left(J_{+}^{\prime}+\lambda f_{+}^{\prime}\right)\right] \\
=J_{+}^{\prime} f_{-} J_{-}^{\prime} f_{+}+\lambda\left(f_{+}^{\prime} f_{-}-f_{+} f_{-}^{\prime}\right)
\end{gathered}
$$

From the equation Eq.(A.4) we get $\lambda=\frac{\varphi-J_{+}}{f_{+}}$. Replacing $\lambda$ with its expression in Eq.(A.7), we obtain:

$\dot{\varphi}$

$$
\begin{aligned}
& =J_{+}^{\prime} f_{-} J_{-}^{\prime} f_{+}+\frac{J_{+}\left(f_{+} f_{-}^{\prime}-f_{+}^{\prime} f_{-}\right)}{f_{+}}+\frac{\varphi\left(f_{+}^{\prime} f_{-}-f_{+} f_{-}^{\prime}\right)}{f_{+}}=\frac{\psi}{f_{+}} \\
& +\frac{\varphi\left(f_{+}^{\prime} f_{-}-f_{+} f_{-}^{\prime}\right)}{f_{+}}
\end{aligned}
$$

with

$$
\psi=J_{+}^{\prime} f_{-} f_{+}-J_{-}^{\prime} f_{+}^{2}+J_{+}\left(f_{+} f_{-}^{\prime}-f_{+}^{\prime} f_{-}\right)
$$

Then, from the previous equation (Eq.(A.8)) we conclude that a necessary condition for having a singular arc on a time interval $\left[t_{1}, t_{2}\right]$ is to have $\psi(m)=0$. Now, let us make the following assumption:

$\mathrm{H} 1$. The function $\psi$ admits a unique positive root $\bar{m}$ and one has

$$
\psi(m)(m-\bar{m})>0 \text { for any } m \neq \bar{m}
$$

Under this condition, $m=\bar{m}$ is thus the unique possible singular arc.

On the singular arc, the constant control $\bar{u}$ to be applied is derived from Eq.(A.2) for $\dot{m}$ $=0$ when $m=\bar{m}$ :

$$
\bar{u}=-\frac{f_{-}(\bar{m})}{f_{+}(\bar{m})} \quad ; \bar{u} \in[-1,1]
$$

As previously, it is optimal to finish with a filtration cycle $(u=1)$. Therefore, the optimal trajectory has to leave the singular $\operatorname{arc}(\bar{m})$ with $u=1$ at a certain switching time $\bar{T}<T$. The switching time $\bar{T}$ can be obtained by integrating backwards the filtration dynamic $f_{1}$ between the final time $T$ and the instant $\bar{T}$, which amounts to writing:

$$
\bar{T}=T-\int_{\bar{m}}^{\bar{m}(T)} \frac{d m}{f_{1}(m)}
$$


where $\bar{m}_{T}$ can be determined from Eq. (A.12) below. On the singular arc, the switching function $\varphi$ is equal to zero. Consequently, we have $H=\bar{\lambda} f_{-}(\bar{m})+J_{-}(\bar{m})$. Moreover, since the optimal trajectory has to leave the singular arc with $u=1$ at a certain switching time $\bar{T}<T$, and at the final time $T$ we have $\lambda(T)=0$, the Hamiltonian at time $T$ is thus given by $H=J(m(T))$. Then, the final state $\bar{m}_{T}=m(T)$ is the solution of:

$$
J\left(\bar{m}_{T}\right)=\bar{\lambda} f_{-}(\bar{m})+J_{-}(\bar{m})=-\frac{J_{+}(\bar{m}) f_{-}(\bar{m})}{f_{+}(\bar{m})}+J_{-}(\bar{m})
$$

Now, let us check the behavior of the optimal trajectory before it reaches the singular arc. As we made the assumption that $\psi$ admits a single positive root, then one can straightforwardly check that:

$$
\left\{\begin{array}{l}
\psi(m)>0 \text { when } m>\bar{m} \\
\psi(m)<0 \text { when } m<\bar{m}
\end{array}\right.
$$

Then the optimal solution is such that:

- if we have $\varphi=0$ with $m<\bar{m}$, then $\dot{\varphi}<0$. This implies that $\varphi$ can change its sign only when decreasing. Therefore, only a control $u=1$ (filtration) can be optimal in the domain $\{m<\bar{m}\}$;

- if we have $\varphi=0$ with $m>\bar{m}$, then $\dot{\varphi}>0$. This implies that $\varphi$ can change its sign only when increasing. Therefore, only a control $u=-1$ (backwash) can be optimal in the domain $\{m>\bar{m}\}$.

Remark: Indeed, it can been shown that when the proposed feedback does not allow to reach $m=\bar{m}$ before the time $\bar{T}$, this optimal feedback is not always optimal: one may have to anticipate the switching and to switch to full filtration along a "switching curve". Nevertheless, these situations occur only when the initial quantity of attached mass is too high, which never occurs in practice. Therefore we do not consider these situations in this work: we restrict our attention to initial conditions such that the time necessary for attaining $m(t)=\bar{m}$ using either filtration or backwash is less than $\bar{T}$.

As an application, we consider the following filtration model:

Mass accumulation dynamics: $f_{1}(m)=\frac{b}{e+m}$

Mass detachment dynamics: $f_{2}(m)=a_{1} m$

Variation dynamics of permeate flux: $J(m)=\frac{d}{e+m}$ 
By replacing the different functions by their expressions in the equation Eq.(A.9), we can calculate its roots and then check the existence of a singular arc. A straightforward computation of the function $\psi$ gives:

$$
\psi(m)=-\frac{1}{4} \frac{a_{1}\left(-a_{1} d m^{2}+J_{B W} b e+2 J_{B W} b m+b d\right)}{(e+m)^{2}}
$$

Therefore, the function $\psi$ vanishes at two different points:

$\bar{m}_{1}=\frac{J_{B W} b+\sqrt{J_{B W}^{2} b^{2}+a_{1} b d^{2}+J_{B W} a_{1} b d e}}{a_{1} d} \quad$ and $\quad \bar{m}_{2}=\frac{J_{B W} b-\sqrt{J_{B W}^{2} b^{2}+a_{1} b d^{2}+J_{B W} a_{1} b d e}}{a_{1} d}$

$\bar{m}_{1}$ is positive. $\bar{m}_{2}$ is negative when $a_{1} b d^{2}+J_{B W} a_{1} b d e>0$ which is always the case. A further computation of the derivative enables us to conclude that $\psi(m)$ is a positive increasing function as $m \geq \bar{m}$. Therefore, assumption $\mathrm{H} 1$ is fulfilled and $\bar{m}_{1}$ is the unique singular arc.

On the singular arc, the constant control $\bar{u}$ to be applied is:

$$
\bar{u}=\frac{a_{1} e \bar{m}+a_{1} \bar{m}^{2}-b}{a_{1} e \bar{m}+a_{1} \bar{m}^{2}+b}
$$

and the final switching time to filtration is given by:

$$
\bar{T}=T-\left[\frac{e\left(\bar{m}_{T}-\bar{m}\right)+0.5\left(\bar{m}_{T}-\bar{m}\right)^{2}}{b}\right]
$$

\section{Appendix B. Case \#2: Operating with variable backwash flux}

In this case, the net water production per membrane area to be maximized can be written as:

$$
L_{T}=\int_{0}^{T} u(t) J(m(t)) d t
$$

where $J(m)$ is the forward or backward permeate flux. The constraint of the problem and the assumptions made on the model functions are the same as in case \#1. The Hamiltonian $H$ and the switching function $\varphi$ for this case can be written as follows: 


$$
\begin{gathered}
H(m, \lambda, u)=\lambda f_{-}(m)+u\left[J(m)+\lambda f_{+}\right] \\
\varphi(m, \lambda)=J(m)+\lambda f_{+}(m)
\end{gathered}
$$

Proceeding in the same way as in case \#1, we found that the optimal solution for case \#2 has the same general structure as in case \#1 (i.e. Eq. (A.5)) but with some noticeable differences in the expressions of the control parameters. In fact, the control parameters $\bar{u}, \bar{T}$ are defined similarly to case \#1 by the Eq. (A.10-11). However, $\bar{m}$ in this case is the only positive root of the function:

$$
\psi=J f_{-} f_{+}+J\left(f_{+} f_{-}^{\prime}-f_{+}^{\prime} f_{-}\right)
$$

and the final state $\bar{m}_{T}=m(T)$ is the solution of:

$$
J\left(\bar{m}_{T}\right)=\bar{\lambda} f_{-}(\bar{m})=-\frac{J_{+}(\bar{m}) f_{-}(\bar{m})}{f_{+}(\bar{m})}
$$

Now, we consider the following model as an application of case \#2:

* Mass accumulation dynamics: $f_{1}(m)=\frac{b}{e+m}$

Mass detachment dynamics: $f_{2}(m)=a_{2} \frac{d}{e+m} m$

Variation dynamics of permeate flux: $J(m)=\frac{d}{e+m}$

A straightforward computation of the function $\psi$ gives:

$$
\psi(m)=\frac{1}{4} \frac{d\left(a_{2}{ }^{2} d^{2} m^{2}-2 a_{2} b d e-2 a_{2} b d m-b^{2}\right)}{(e+m)^{4}}
$$

The function $\psi$ admits of two possible roots:

$$
\bar{m}_{1}=\frac{b+\sqrt{2 b^{2}+2 a_{2} b d e}}{a_{2} d} \text { and } \bar{m}_{2}=\frac{b-\sqrt{2 b^{2}+2 a_{2} b d e}}{a_{2} d}
$$


$\bar{m}_{2}$ is negative when $b^{2}+2 a_{2} b d e>0$ which is always true because $a_{2}, \mathrm{~b}, \mathrm{~d}$ and e are positive variables. Thus, assumption $\mathrm{H} 1$ is fulfilled and we conclude that $\bar{m}_{1}$ is the unique singular arc.

In this case, the constant control to be applied on the singular arc has the following analytical form:

$$
\bar{u}=\sqrt{2} \frac{\sqrt{b\left(a_{2} d e+b\right)}}{2 b+\sqrt{2} \sqrt{b\left(a_{2} d e+b\right)}}
$$

and the final switching time, $\bar{T}$, is expressed as in the Eq.(A.16). 

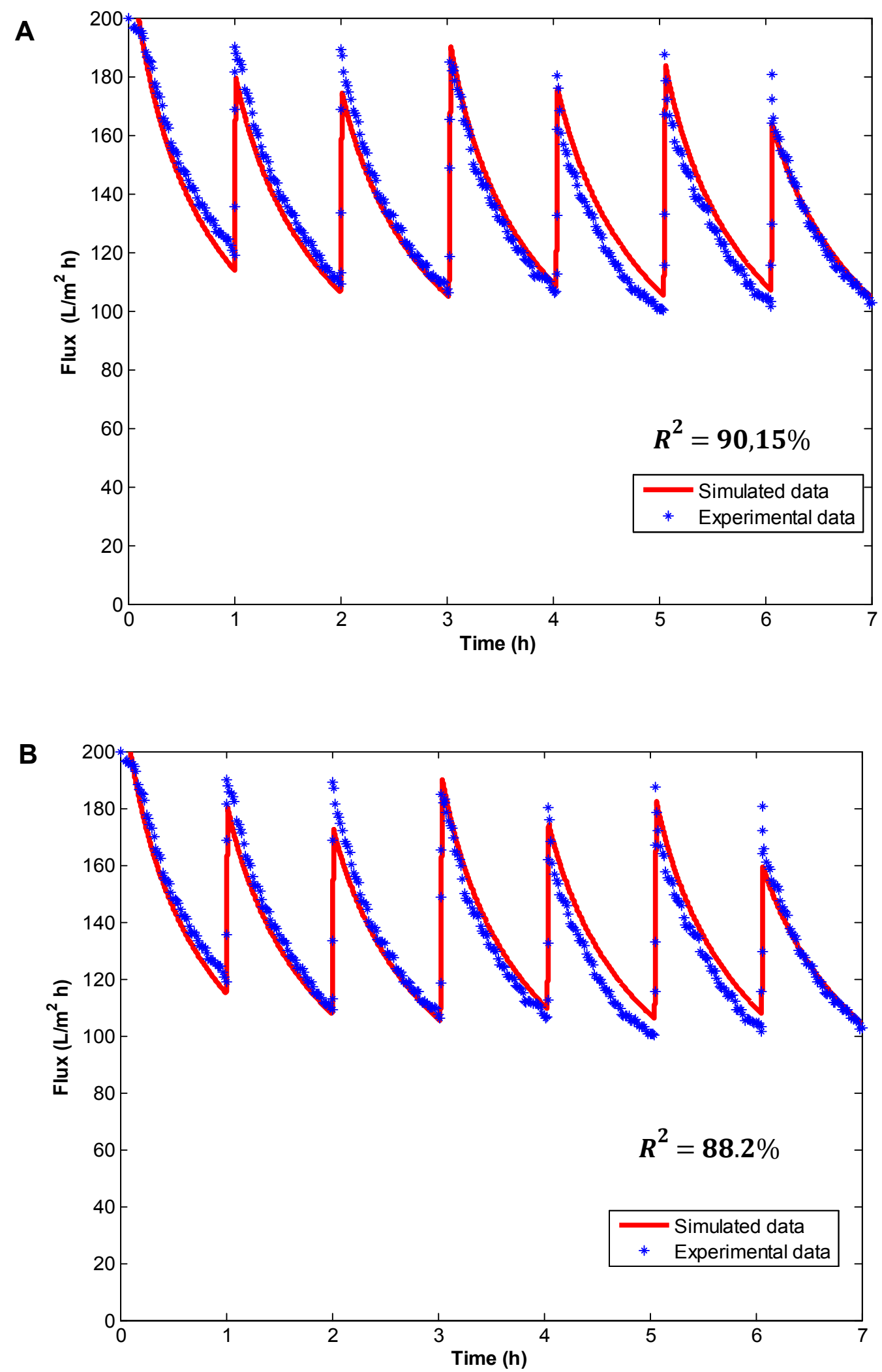

Figure 
1: Model fitting with experimental data obtained from the literature and represented by blue stars in case \#1 (constant backwash flux) (A) and in case \#2 (B) (variable backwash flux)

A

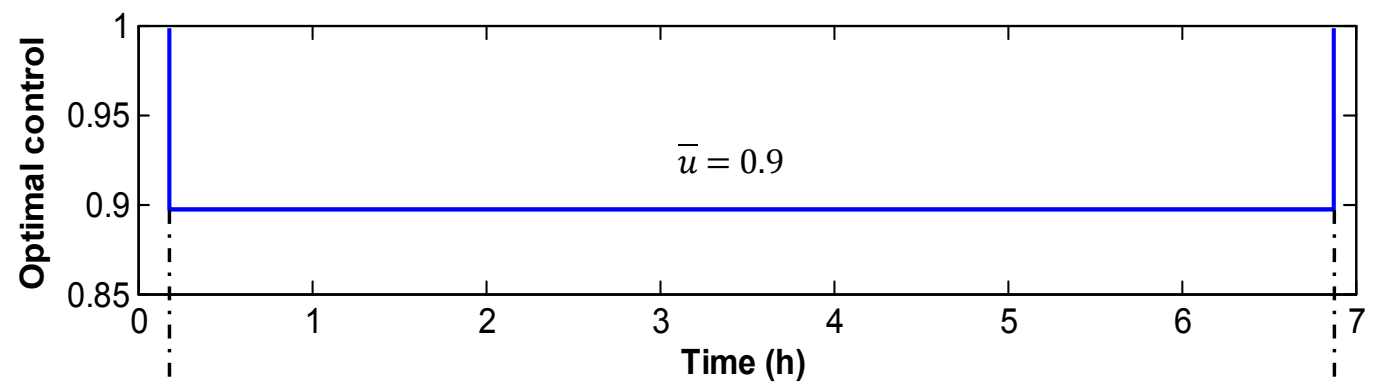

B
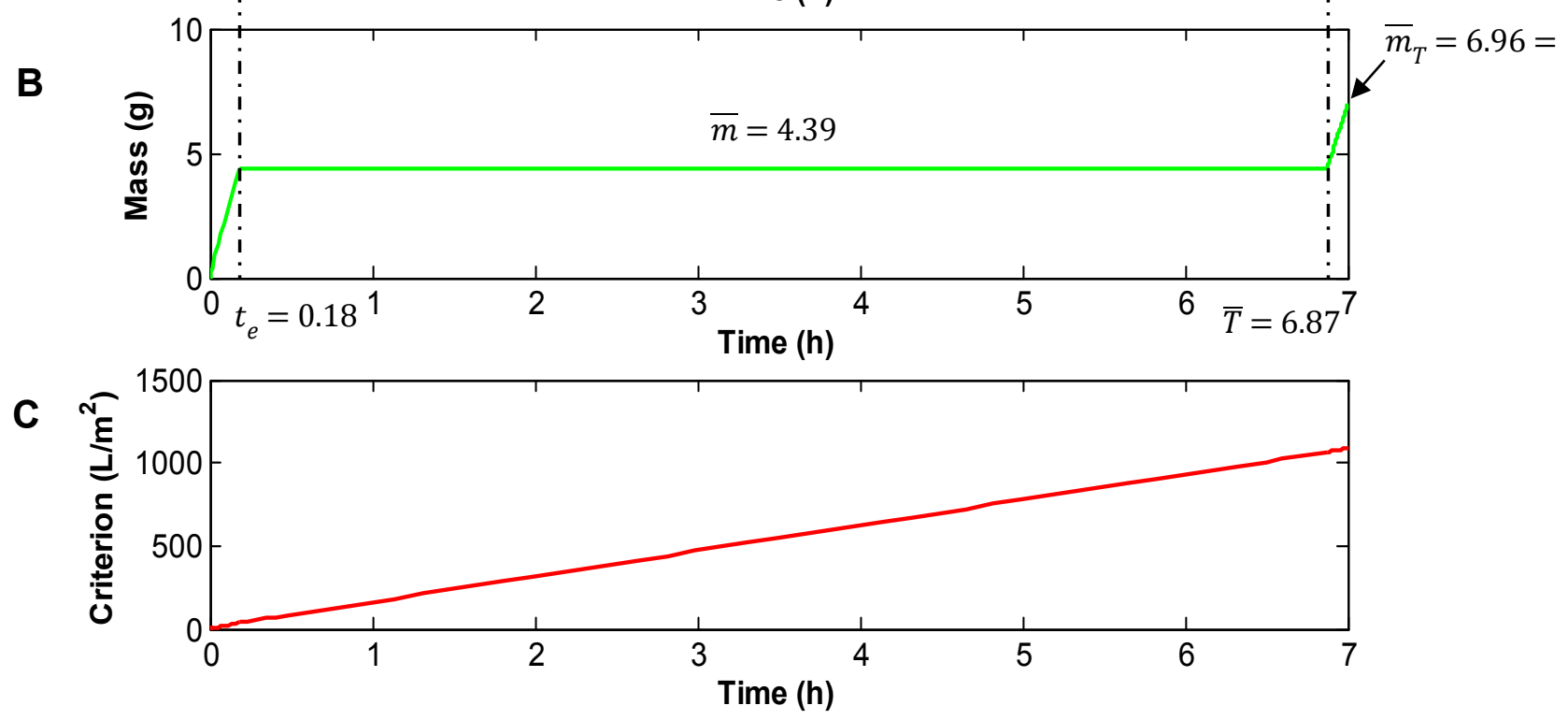

Figure 2: Case \#1 (constant backwash flux) (A) The theoretical optimal operating strategy over T; (B) The corresponding mass accumulated on the membrane surface over T; (C) The corresponding volume produced over $\mathrm{T}$ 

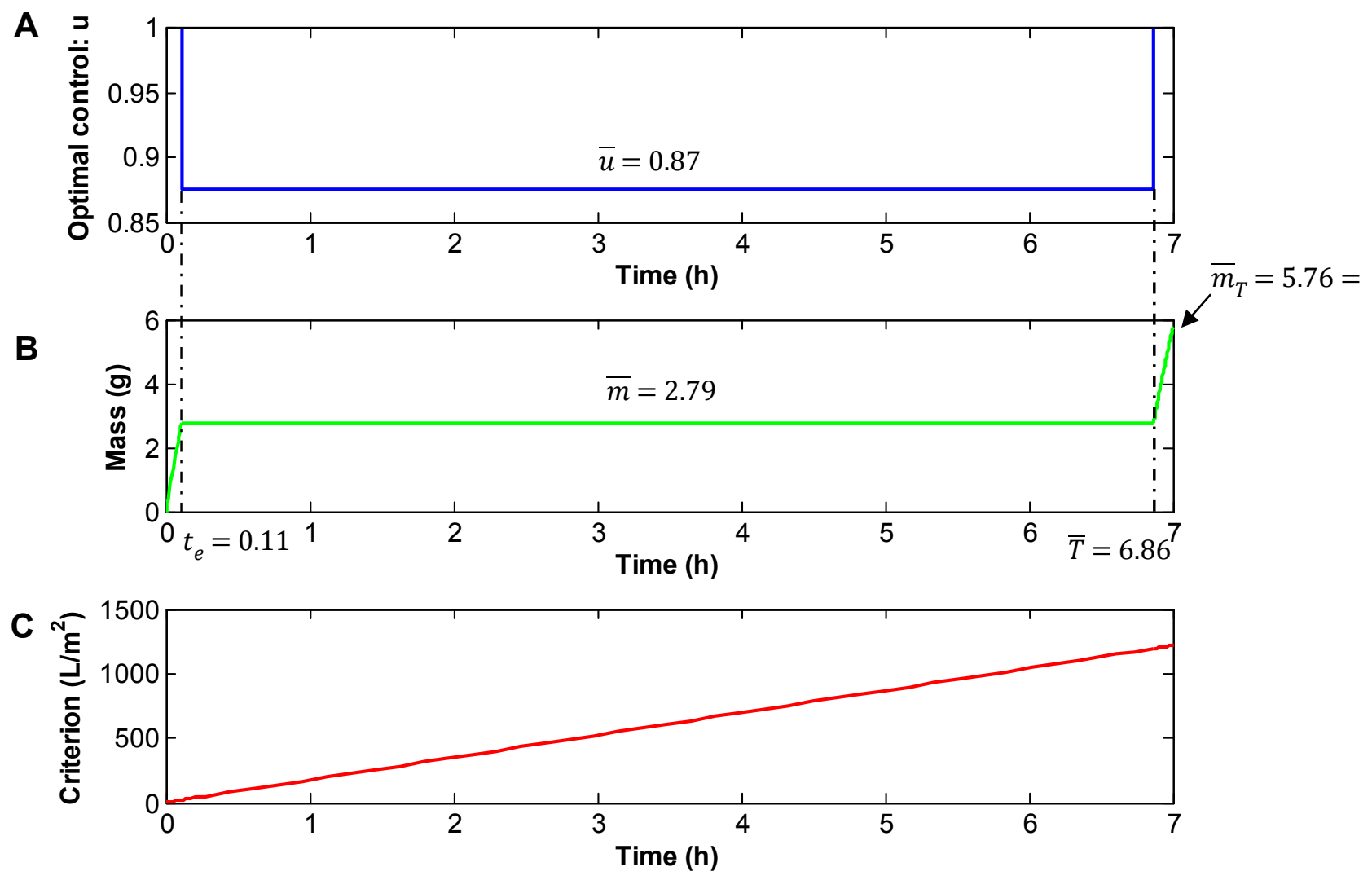

Figure 3: Case \#2 (variable backwash flux) (A) The theoretical optimal operating strategy over T; (B) The corresponding mass accumulated on the membrane surface over T; (C) The corresponding volume produced over $\mathrm{T}$ 


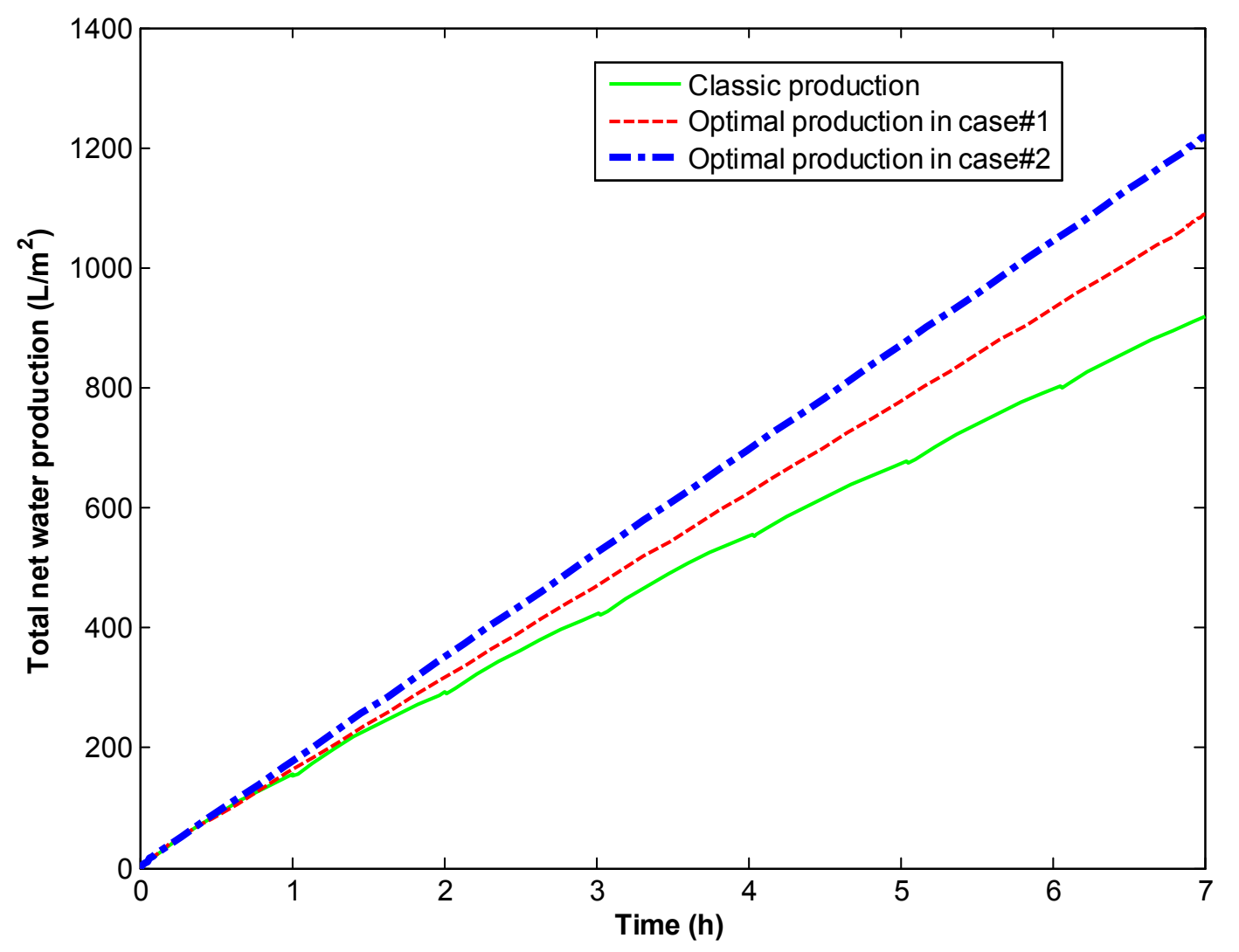

Figure 4: Comparison of the net volume produced per membrane area over time of the published strategy (solid green line); the optimal strategy in case \#1 (constant backwash flux) (dashed red line); and in case \#2 (variable backwash flux) (dash-dot blue line). 

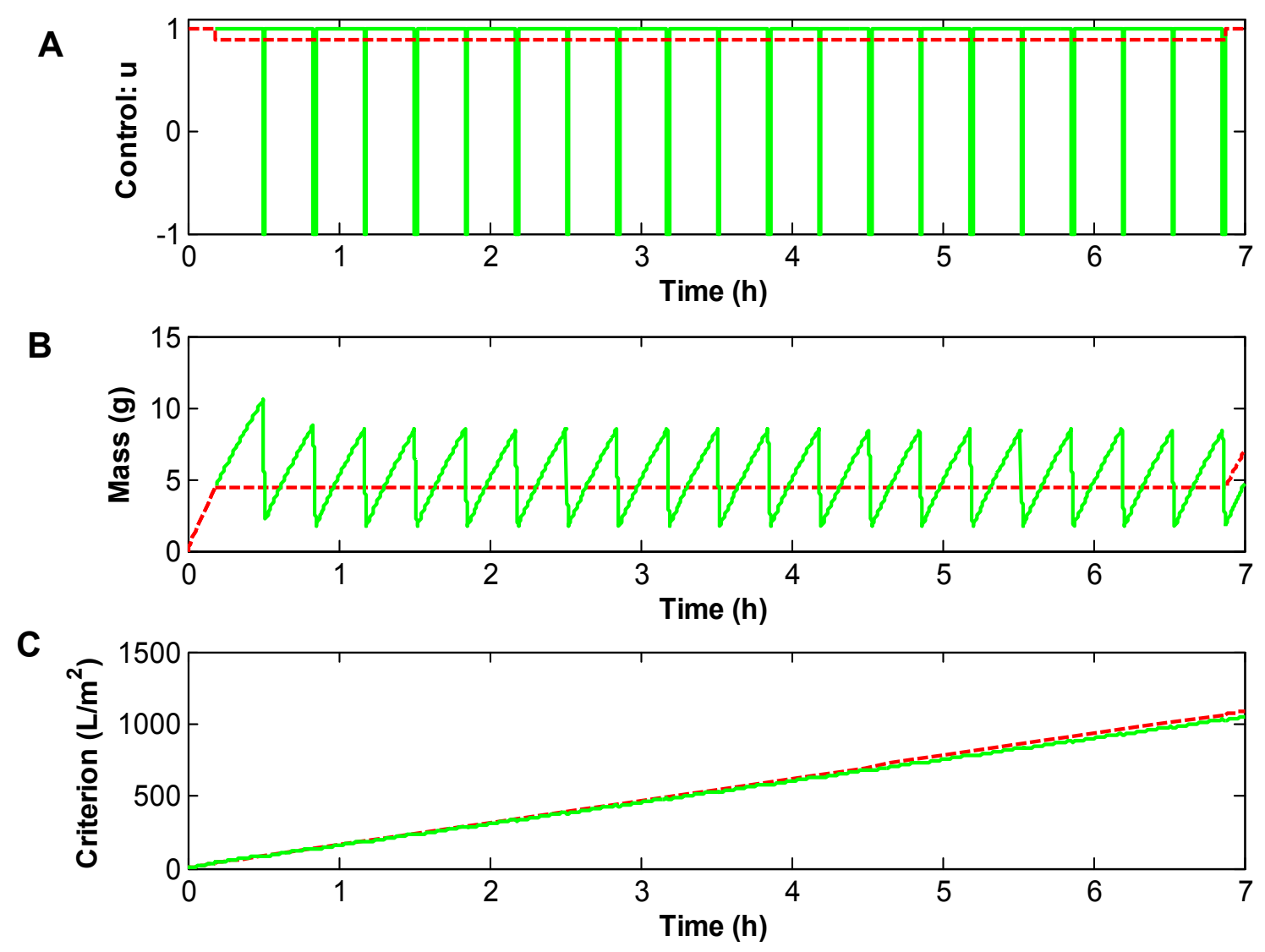

Figure 5: Case \#1(constant backwash flux) Example of the adapted strategy for a number of cycles $\mathrm{N}=20$ to be carried in the singular arc. The dashed red line corresponds to the theoretical optimal strategy, the solid green line corresponds to the adapted strategy that approximates the optimal control solution. (A) The control sequence over T; (B) The cake layer mass on the membrane surface over T; (C) The net volume produced per membrane area over $\mathrm{T}$. 


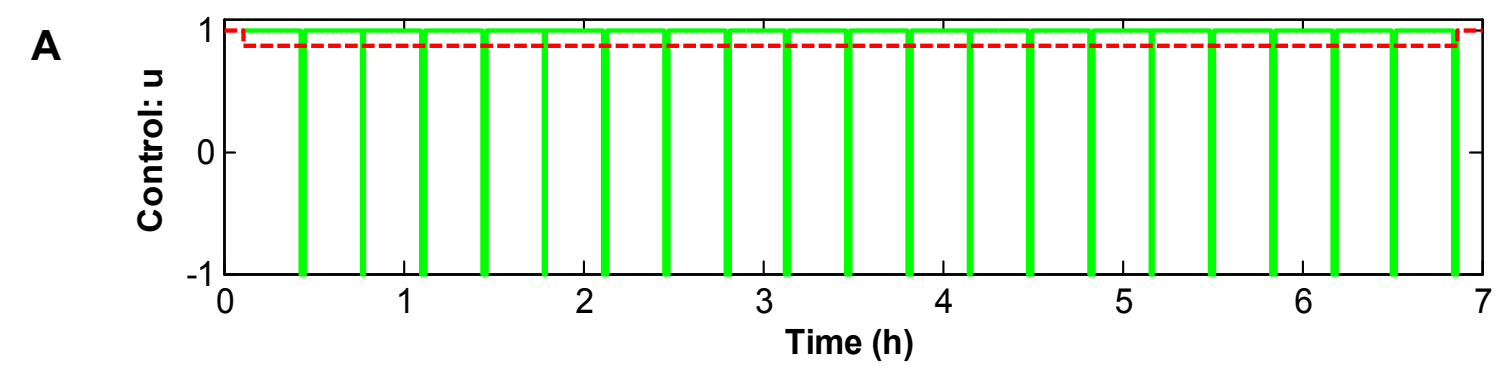

B

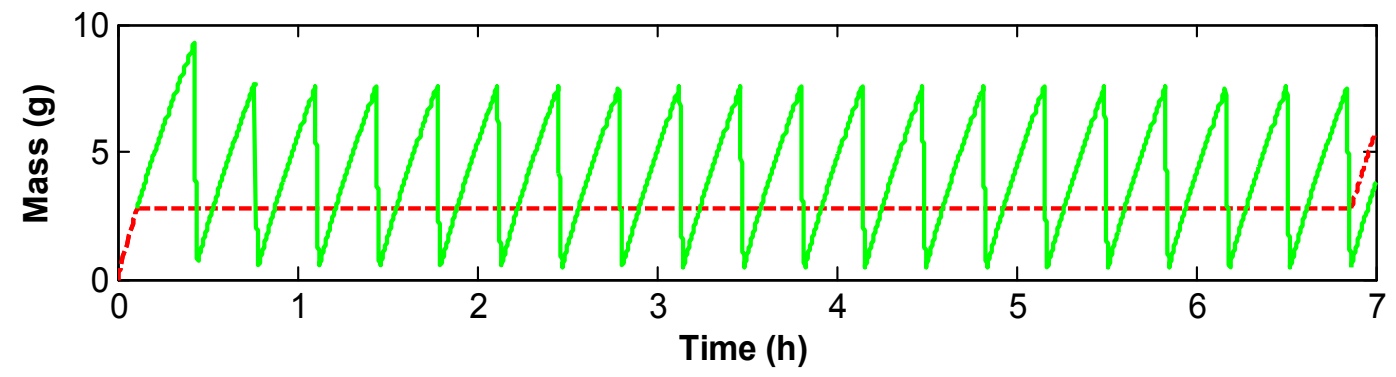

C

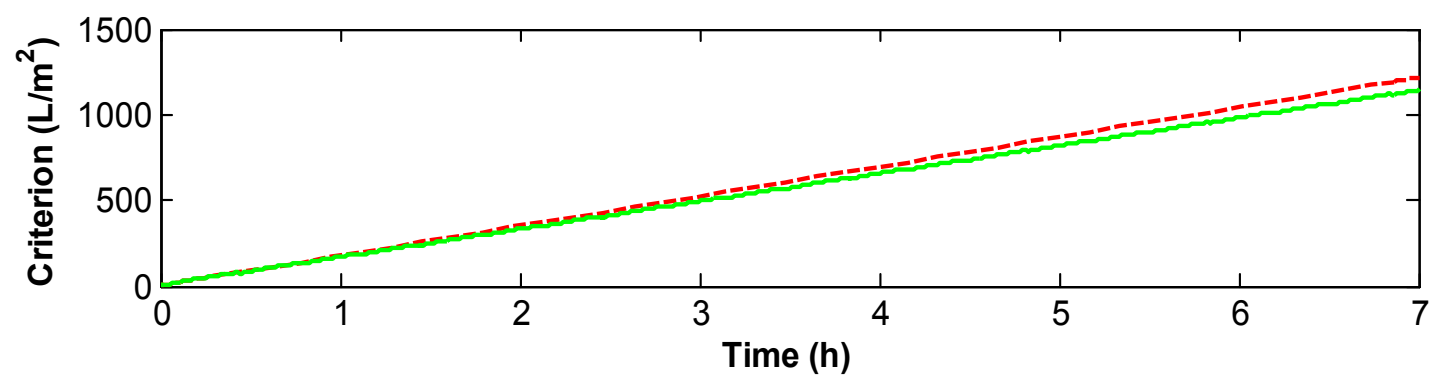

Figure 6: Case \#2 (variable backwash flux) Example of the adapted strategy for a number of cycles $\mathrm{N}=20$ to be carried in the singular arc. The dashed red line corresponds to the theoretical optimal strategy, the solid green line corresponds to the adapted strategy that approximates the optimal control solution. (A) The control sequence over T; (B) The cake layer mass on the membrane surface over T; (C) The net volume produced per membrane area over $\mathrm{T}$. 
A

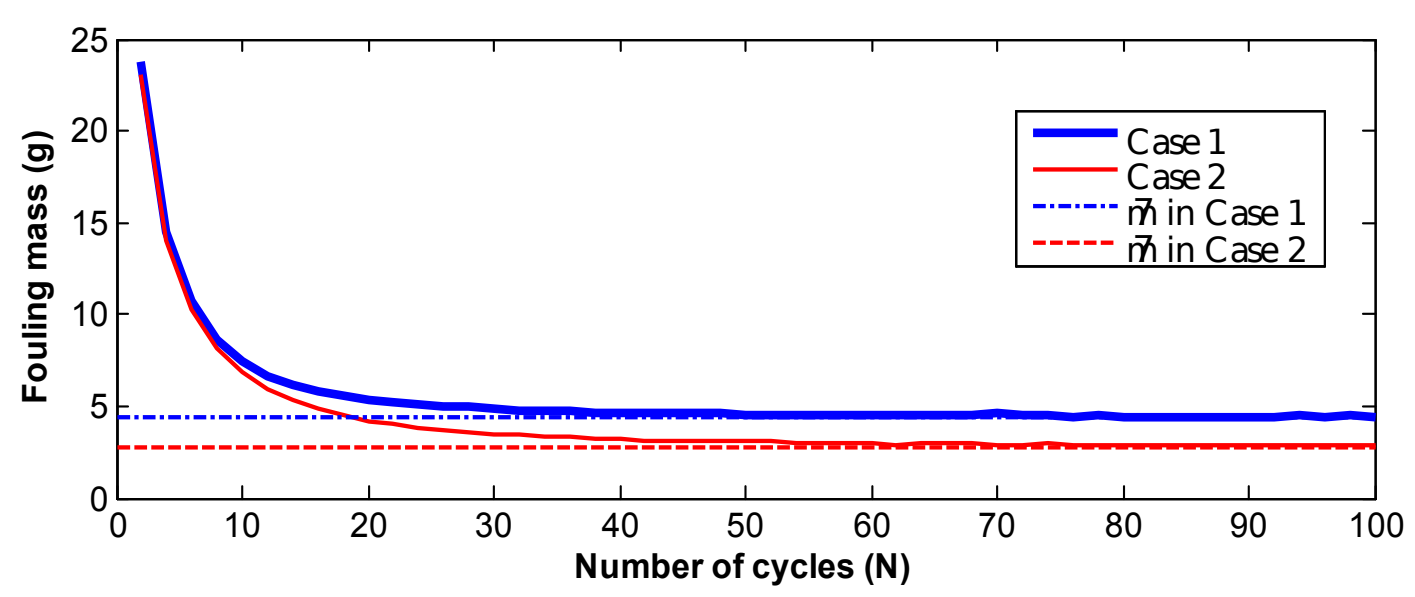

B

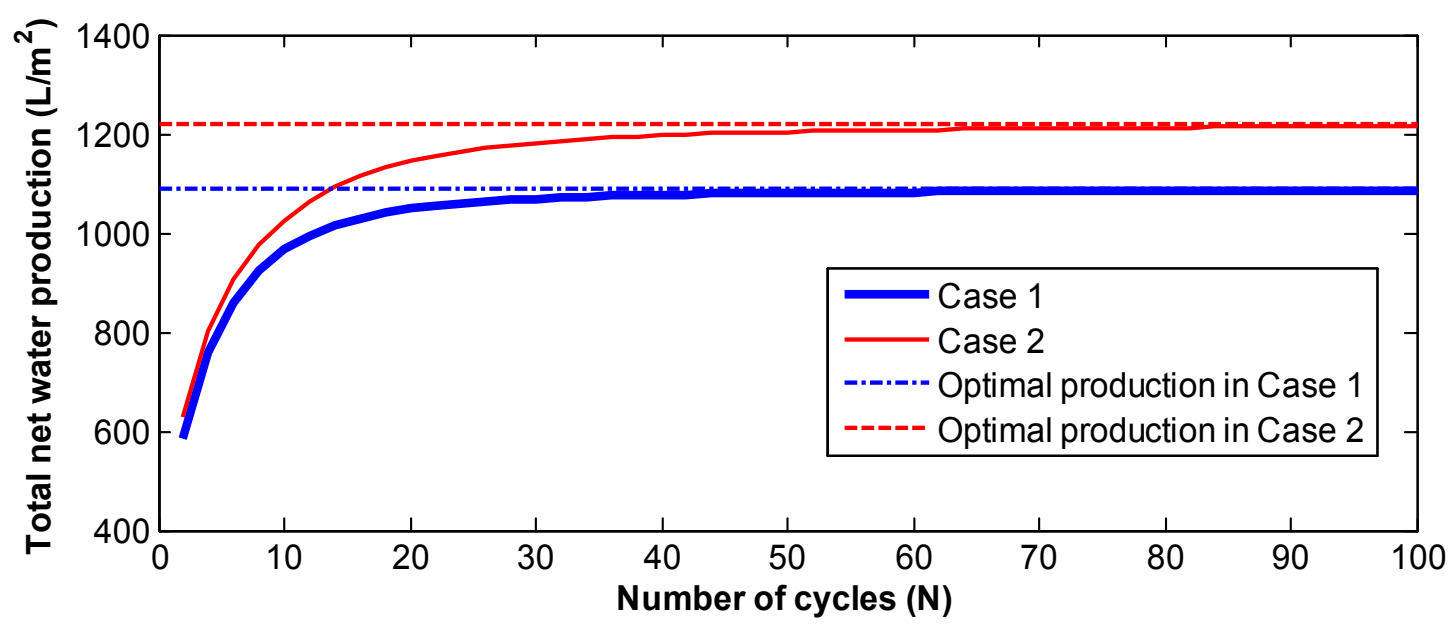

Figure 7: (A) The evolution of the mean mass accumulated on the membrane surface as a function of $\mathrm{N}$ over a 7-hours operating period with respect to the number of backwash cycles $\mathrm{N}$ applied on the singular arc; (B) The evolution of the net production per membrane area of a membrane filtration process over a 7-hour operating period with respect to the number of backwashing cycles $\mathrm{N}$ applied to the singular arc. 


\section{CLOSED-LOOP SYSTEM}

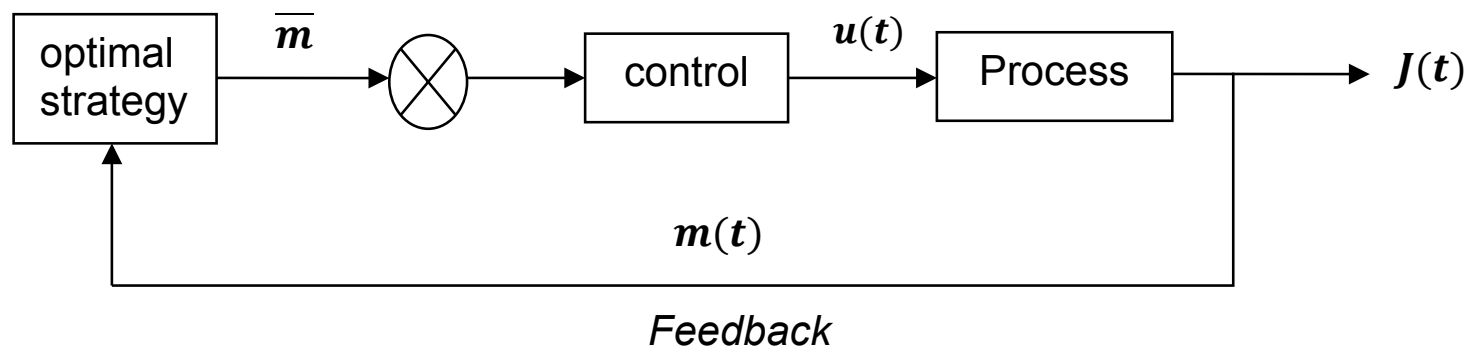

OPEN-LOOP SYSTEM

\begin{tabular}{|c|}
\hline optimal \\
\hline
\end{tabular}

Figure 8: Closed-loop and open-loop controller 
Table 1: Physical significance of the model parameters in cases \#1(constant backwash flux) and \#2 (variable backwash flux)

\begin{tabular}{ccc}
\hline Model parameter & Expression & Unit \\
\hline$b$ & $\frac{T M P \cdot A^{2} \cdot C_{\text {tot }}}{\mu \cdot \alpha}$ & $g^{2} \cdot h^{-1}$ \\
\hline$e$ & $\frac{R_{0} A}{\alpha}$ & $g$ \\
\hline$d$ & $T M P \cdot \frac{A}{\mu \cdot \alpha}$ & $g \cdot m \cdot h^{-1}$ \\
\hline$a_{1}$ & $\omega_{B W} J_{B W}$ & $h^{-1}$ \\
\hline$a_{2}$ & $\omega_{B W}$ & $m^{-1}$
\end{tabular}

${ }^{{ }^{\prime} \mu}$ is the permeate viscosity (Pa.s)

Table 2: Parameter values used in this study that best reproduce the permeate flux dynamic published in [1]

\begin{tabular}{ccc}
\hline Model parameter & Value & Unit \\
\hline$b$ & 490.07 & $g^{2} \cdot h^{-1}$ \\
\hline$e$ & 18.06 & $g$ \\
\hline$d$ & 4125.6 & $g \cdot m \cdot h^{-1}$ \\
\hline$a_{1}$ & 91.7 & $h^{-1}$ \\
\hline$a_{2}$ & 0.64 & $m^{-1}$ \\
\hline
\end{tabular}


Table 3: Control parameters computed using the identified model

\begin{tabular}{cccc}
\hline Control Parameter & Case \#1 & Case \#2 & Units \\
\hline $\bar{m}$ & 4.39 & 2.79 & $g$ \\
\hline $\bar{u}$ & 0.9 & 0.87 & - \\
\hline $\bar{m}_{T}$ & 6.96 & 5.76 & $g$ \\
\hline$t_{e}$ & 0.18 & 0.11 & $h$ \\
\hline $\bar{T}$ & 6.87 & 6.86 & $h$ \\
\hline
\end{tabular}

Table 4: Robustness study. Mean minimum and maximum deviations $(\gamma)$ of the process production per membrane area from the optimum based on 1000 iterations for different biased values of the model's parameters.

\begin{tabular}{ccccc}
\hline \multirow{2}{*}{ Biased model parameters } & \multicolumn{2}{c}{ Minimum $\gamma(\%)$} & \multicolumn{2}{c}{ Maximum $\gamma(\%)$} \\
\cline { 2 - 4 } & Case \#1 & Case \#2 & Case \#1 & Case \#2 \\
\hline$b \pm 20 \% b$ & -3.52 & -2.61 & 3.97 & 2.8 \\
\hline$e \pm 20 \% e$ & -13.6 & -14.65 & 17.06 & 20.76 \\
\hline$a_{k} \pm 20 \% a_{k}$ & -4.02 & -3.09 & 3.05 & 2.19 \\
\hline$d \pm 20 \% d$ & -22.5 & -22.47 & 22.5 & 22.6 \\
\hline${ }^{*} a_{k}$ denotes $a_{1}(i . e . k=1)$ in case \#1(constant backwash flux) and $a_{2}($ i.e. $k=2)$ in case \\
\#2 (variable backwash flux)
\end{tabular}


